\title{
ANÁlisis PALINOLÓGICO DE MIELES Y CARGAS DE POLEN DE APIS Mellifera (APIDAE) DE LA REGIÓN CENTRO Y NORTE DEL ESTADO de Guerrero, México
}

\author{
Elia Ramírez-Arriaga ${ }^{1,4}$, Angélica Martínez-Bernal ${ }^{2}$, Nadia Ramírez Maldonado 3 \\ y EnRIQUe MartíneZ-HeRnándeZ ${ }^{1}$ \\ 'Laboratorio de Palinología, Departamento de Paleontología, Instituto de Geología, \\ Universidad Nacional Autónoma de México, Ciudad de México, México \\ ${ }^{2}$ Departamento de Biología, División de Ciencias Biológicas y de la Salud, \\ Universidad Autónoma Metropolitana-Iztapalapa, Ciudad de México, México. \\ ${ }^{3}$ Universidad Autónoma Metropolitana-Xochimilco, Ciudad de México, México \\ ${ }^{4}$ Autor para la correspondencia: elia@unam.mx
}

\begin{abstract}
Resumen: El presente trabajo es una contribución al conocimiento del contenido polínico de seis muestras de miel y seis muestras de cargas de polen de Apis mellifera para conocer el origen botánico y geográfico, procedentes de las regiones Centro y Norte de Guerrero; ambas zonas de interés apícola, durante los meses de noviembre-diciembre del 2005 y marzo del 2006. Asimismo, se analiza el comportamiento de recolecta de la abeja melífera. Los análisis melisopalinológicos mostraron un total de 43 tipos polínicos pertenecientes a 27 familias, solamente 15 taxones de la flora nativa fueron consideradas de importancia y sus descripciones palinológicas son incluidas: Cosmos sulphureus, Dyssodia papposa, Taraxacum campylodes, Tithonia tubaeformis, Vernonia sp. (Asteraceae), Coutoubea sp. (Gentianaceae), Dalea sp., Leucaena leucocephala (Fabaceae), Quercus sp. (Fagaceae), Miconia sp. (Melastomataceae), Pisonia sp. (Nyctaginaceae), Dodonaea viscosa, Paullinia sp. (Sapindaceae), Solanum sp. (Solanaceae) y Heliocarpus donnellsmithii (Malvaceae), así como la planta cultivada Zea mays (Poaceae). Se caracterizaron, dos mieles como oligoflorales y cuatro multiflorales. El índice de diversidad en las muestras de miel varió de 1.7 a 2.3, y en las cargas de polen de 1.6 a 2.1. El índice de uniformidad en la miel varió de 0.8 a 0.9 , mientras que en las cargas de polen se encontraron en el intervalo de 0.6 a 0.9. La abeja Apis mellifera tuvo un comportamiento poliléctico, el estrato herbáceo fue mejor explotado, seguido del arbustivo y arbóreo.
\end{abstract}

Palabras clave: abeja melífera, melisopalinología, miel oligofloral y multifloral, morfología polínica, región apícola.

\begin{abstract}
The present work is a contribution to knowledge of polen recovered from six Apis mellifera honey samples and six pollen loads samples, in order to characterize their botanical and geographical origin. Samples were collected from central and northern Guerrero -both regions of beekeeping importance- during November-December 2005 and March 2006. Honeybee foraging behavior was also analyzed. Melissopalynological results showed a total of 43 pollen types belonging to 27 families; only 15 taxa of native flora were considered of importance, and their palynological descriptions are included: Cosmos sulphureus, Dyssodia papposa, Taraxacum campylodes, Tithonia tubaeformis, and Vernonia sp. (Asteraceae), Coutoubea sp. (Gentianaceae), Dalea sp. and Leucaena leucocephala (Fabaceae), Quercus sp. (Fagaceae), Miconia sp. (Melastomataceae), Pisonia sp. (Nyctaginaceae), Dodonaea viscosa and Paullinia sp. (Sapindaceae), Solanum sp. (Solanaceae), and Heliocarpus donnellsmithii (Malvaceae) as well as Zea mays (Poaceae), an abundant crop. Two oligofloral and four multifloral honey samples were characterized. Diversity indexes varied from 1.7 to 2.3 in honey samples, while the pollen loads registered 1.6 to 2.1 values in diversity, the evenness index in honey samples varied from 0.8 to 0.9 , whereas pollen load samples registered a range from 0.6 to 0.9 . Apis mellifera showed polylectic behavior; the herbaceous stratum was the most foraged, followed in importance by tree and shrub strata.
\end{abstract}

Key words: honeybee, melissopalynology, oligofloral and multifloral honey, pollen morphology, beekeeping region.

a gran diversidad florística y climática de México son

factores que han desencadenado la producción de miel con una gran variedad de sabores, aromas y colores, características que en conjunto se denominan propiedades orga- nolépticas (Martínez-Hernández y Ramírez-Arriaga, 1998). La procedencia floral del néctar -a partir del cual las abejas elaboran la miel- y de las cargas de polen -fuente importante de proteínas para la colonia-, puede conocerse me- 
diante análisis palinológicos. En este sentido, los estudios melisopalinológicos permiten no sólo caracterizar y conocer el origen botánico y geográfico de la miel y cargas de polen, sino del alimento larval así como de las resinas o propóleos (Crane, 1975; Rivero et al., 2003).

En la década de los ochenta, se iniciaron los estudios melisopalinológicos en zonas tropicales de México, enfocados en Apis mellifera L. (Lobreau-Callen y Callen, 1982; Cárdenas-Chávez, 1985; Villanueva, 1984; Alvarado y Delgado, 1985; Villanueva, 2002). Sin embargo, no fue sino a partir de la década de los noventa cuando estas investigaciones se incrementaron notablemente, abarcando a las abejas melíferas y a las abejas nativas (melipónidos), realizándose análisis muy sistemáticos, algunos de ellos durante ciclos anuales (Martínez-Hernández et al., 1993; Ramírez-Arriaga et al., 1995), mientras que otros se encaminaron al análisis de mieles producidas en las épocas importantes de cosecha, en algunos municipios o distritos de varios estados de la República Mexicana (Martínez-Hernández y Ramírez-Arriaga, 1998; Ramírez-Arriaga y Martínez-Hernández, 2007; Navarro, 2008; Díaz, 2008; Castellanos-Potenciano, 2010; Quiroz-García et al., 2011; Ramírez-Arriaga et al., 2011).

Además, se han publicado trabajos comparativos sobre el comportamiento de recolecta de Apis mellifera y abejas sin aguijón (Ramírez-Arriaga y Martínez-Hernández, 2007), contrastando con aquellas investigaciones exclusivas en abejas nativas solitarias y comunales (Ramírez-Arriaga, 1989; Ramírez-Arriaga y Martínez-Hernández, 1998; Quiroz-García y Palacios-Chávez, 1999).

En síntesis, los estudios melisopalinológicos en Apis mellifera y melipónidos se han concentrado en varias regiones de México: (1) sur y sureste: en Oaxaca (Navarro, 2008; Díaz, 2008; Ramírez-Arriaga et al., 2011), y Chiapas (Martínez-Hernández et al.,1993); (2) península de Yucatán: en los estados de Campeche (Pacheco-Palomo, 2011), Yucatán y Quintana Roo (Villanueva, 2002); (3) Golfo de México: en Veracruz (Villanueva, 1984), Tabasco (Castellanos-Potenciano, 2010; Castellanos-Potenciano et al., 2012) y (4) centro: en Morelos (Quiroz-García y Arreguín-Sánchez, 2008), Puebla (Ramírez-Arriaga y Martínez-Hernández, 2007), el Distrito Federal (Piedras-Gutiérrez y Quiroz García, 2007) y Zacatecas (Acosta-Castellanos et al., 2011). Es importante destacar, que en un país como México, donde la actividad apícola es de gran relevancia a nivel nacional, existe la necesidad de caracterizar las mieles por medio de su contenido polínico como un medio de control de calidad y profundizar en el estudio polínico como se ha venido realizando en Europa (Downey et al., 2005; Rodríguez de la Cruz et al., 2013). Además, debido a que en nuestro país se producen grandes cantidades de mieles monoflorales (con un taxón dominante $\geq 45 \%$ ) y multiflorales, recientemente se propuso una nueva clasificación de las segundas: (a) las oligoflorales dominadas por dos o más taxones de una familia de plantas con 16-45\%, (b) biflorales, con dos taxones relevantes de diferentes familias botánicas presentes del 16 al $45 \%$ y (c) las estrictamente multiflorales, con tres o más taxones de diferentes familias con porcentajes $\geq$ $10 \%$ (Ramírez-Arriaga et al., 2011). Por otro lado, México se encuentra entre los cinco primeros países productores de miel a escala mundial, después de China, Argentina, Estados Unidos y Turquía; y la miel nacional es bien cotizada en los exigentes mercados internacionales de Alemania, Reino Unido, Arabia Saudita, Suiza, Estados Unidos y Japón; razón por la cual la caracterización de mieles podría darle un valor agregado en beneficio del sector apícola (SAGARPA, 2009, 2015).

La presente investigación se llevó a cabo en el estado de Guerrero, considerado dentro de las diez entidades principales productoras de miel a nivel nacional (Villegas-Durán et al., 2002) y tiene como objetivo analizar palinológicamente muestras de miel y cargas de polen en las regiones geográficas Norte y Centro, ambas de gran interés apícola para el estado, con la finalidad de ampliar el conocimiento sobre la utilización de los recursos florales de Apis mellifera en los períodos importantes de cosecha, lo que permitirá comprender y analizar el comportamiento de recolecta de la abeja melífera; asimismo se caracterizan las mieles en monoflorales, oligoflorales o multiflorales de acuerdo al contenido polínico establecido para mieles mexicanas (Ramírez-Arriaga et al., 2011).

\section{Materiales y métodos}

Área de Estudio. El estado de Guerrero se localiza en el sur de México, y se divide en siete regiones geográficas (GEG, 2016a). Las áreas de muestreo corresponden a dos municipios de interés apícola en dicho estado: Tixtla de Guerrero (región centro) y Teloloapan (región norte) (Figura 1).

Región Centro. En el municipio de Tixtla de Guerrero se visitó un apiario ubicado a $17^{\circ} 32$ ' 48.3 " de latitud norte y $99^{\circ} 15^{\prime} 58.3^{\prime \prime}$ de longitud oeste (Figura 1) a 1,830 m s.n.m. En esta zona predominan tres tipos de clima: el subhúmedo-cálido, subhúmedo-templado y el subhúmedo-semicálido, la temperatura media anual es de $28{ }^{\circ} \mathrm{C}$, con lluvias de mayo a septiembre. Los tipos principales de vegetación son el bosque de pino-encino y la selva baja caducifolia (INEGI, 2005; GEG, 2016b).

Región Norte. El apiario objeto de estudio se localizó en el municipio de Teloloapan a $18^{\circ} 24^{\prime} 0.30^{\prime \prime}$ de latitud norte y $99^{\circ} 53^{\prime} 15.8^{\prime \prime}$ de longitud oeste (Figura 1), a 1,400 m s.n.m. El clima predominante es cálido-subhúmedo, la temperatura media anual es de $25^{\circ} \mathrm{C}$, la precipitación media anual es de 1,200 mm, con lluvias en verano. La vegetación predominante es la selva baja caducifolia (INEGI, 2005; GEG, 2016c).

Muestreo. En la región Centro (Tixtla de Guerrero) y Norte (Teloloapan) se colectaron un total de 12 muestras - seis de miel y seis de cargas de polen-durante los meses de 


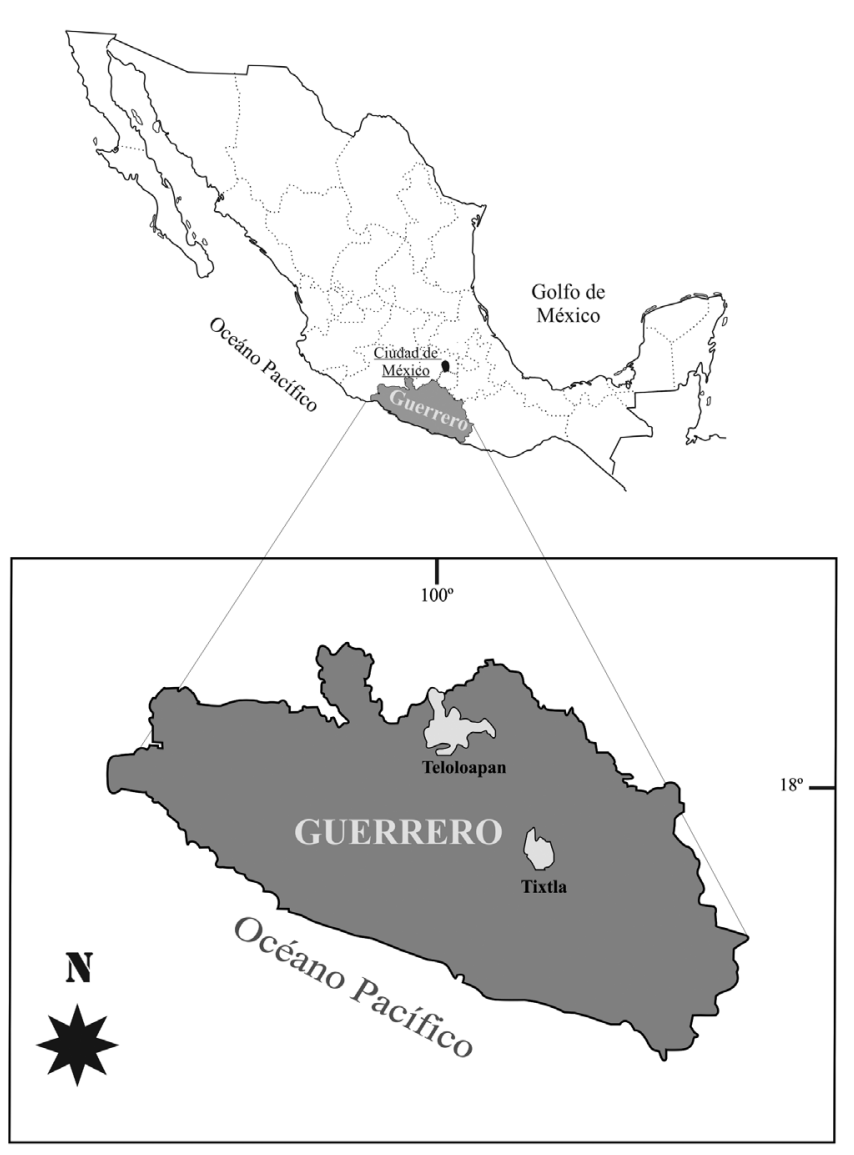

Figura 1. Mapa donde se ubican los municipios de colecta de miel y cargas de polen de Apis mellifera en la zona Centro (Tixtla) y Norte (Teloloapan) del estado de Guerrero.

noviembre y diciembre de 2005 y marzo del 2006, que corresponden a las principales épocas de floración. Además se colectaron ejemplares botánicos de la flora apibotánica, en los alrededores de los apiarios.

La recolección de 100-250 ml de miel se obtuvo mediante el corte de una sección del panal operculado, el cual se colocó en baño maría para separar la miel de la cera. Por otro lado, la toma de muestras de cargas de polen se realizó por medio de trampas de polen colocadas en la base de las colmenas por un período de 2 a 3 días.

Técnica de análisis melisopalinológico. El análisis químico consistió en procesar $50 \mathrm{ml}$ de miel por muestra de acuerdo a Louveaux et al. (1978), mientras que $20 \mathrm{~g}$ de cargas de polen fueron diluidas en $150 \mathrm{ml}$ de agua tibia y homogeneizadas; en ambos casos se realizó la técnica de acetólisis de Erdtman (1960), se elaboraron laminillas permanentes con gelatina glicerinada y se registraron e incorporaron a la colección palinológica reciente del Laboratorio de Paleopalinología, del Instituto de Geología de la Universidad Nacional Autónoma de México (UNAM).

El análisis melisopalinológico consistió en la descripción morfológica de los granos de polen y la determinación a nivel de familia, género y/o especie por comparación con la colección palinológica de referencia del Instituto de Geología de la UNAM y consultando literatura especializada en palinología (Palacios et al., 1991; Vázquez y Martínez, 1991; MartínezHernández et al., 1993; Vargas y Ludlow, 1993; Olvera, 1997; Olivera et al., 1998; Alfaro et al., 2010).

Una vez determinados los tipos polínicos, y con el objetivo $100 \times$ se contaron 500 granos de polen al azar por muestra. Posteriormente, se calcularon los porcentajes de cada taxón en el programa Excel y se graficaron empleando el programa Tilia Graph (Grimm, 2011), lo que facilitó el análisis de los conjuntos palinológicos y se analizó la representatividad de los mismos por muestra. Asimismo, se realizaron las descripciones palinológicas de los taxones de importancia $(\geq$ $10 \%$ ) basadas en los caracteres morfólogicos de 25 granos de polen por taxón y siguiendo la nomenclatura de Punt et al. (2007). Los tipos polínicos más abundantes fueron fotografiados con un microscopio Axio Lab Carl-Zeiss empleando el programa Axio Vision (versión 4.8.2). Por otro lado, se fotografiaron algunos taxones utilizando el microscopio electrónico de barrido del Instituto de Geología de la UNAM marca Jeol JSM-6300 y electrones secundarios para la obtención de imágenes, las condiciones de trabajo fueron $29 \mathrm{kV}$, en modo de alto vacío.

Análisis estadísticos. Se calcularon los índices de diversidad de Shannon-Weaver (1949) y de uniformidad de Pielou (1977) empleando el programa Species Diversity and Richness (Seaby y Henderson, 2007). Además se realizaron análisis comparativos con otras zonas apícolas.

Recolección de material botánico. La recolecta de ejemplares botánicos se realizó en los alrededores de los apiarios de las dos regiones de estudio en noviembre y marzo; se recolectaron 70 ejemplares de acuerdo con los métodos propuestos por Lot y Chiang (1986). El material botánico se determinó utilizando diferentes claves de identificación y se registró e incorporó a la colección científica del Herbario Metropolitano (UAMIZ) de la Universidad Autónoma Metropolitana, Unidad Iztapalapa.

\section{Resultados}

Los análisis melisopalinológicos aplicados a seis muestras de miel y seis de cargas de polen colectadas en las regiones centro y norte del estado de Guerrero mostraron un total de 43 tipos polínicos pertenecientes a 27 familias botánicas. Además, para la muestra de miel M-12511 de la región Norte se realizó un recalculo basado en las especies exclusivamente nectaríferas (Tabla 1).

Región Centro. En las tres muestras de miel analizadas se registraron un total de 30 taxones pertenecientes a 20 familias, siendo el mes de marzo el de mayor riqueza con 26 taxones observados, mientras que en los meses de noviembre y diciembre se registraron sólo diez taxones. Las cargas de polen analizadas para la misma región, revelaron un total de 
Elia RamíreZ-Arriaga ET AL.

Tabla 1. Porcentajes de taxones registrados en la miel $(\mathbf{M})$, recalculo basado únicamente en especies nectaríferas (EN) y cargas de polen $(\mathbf{P})$ de Apis mellifera en Guerrero.

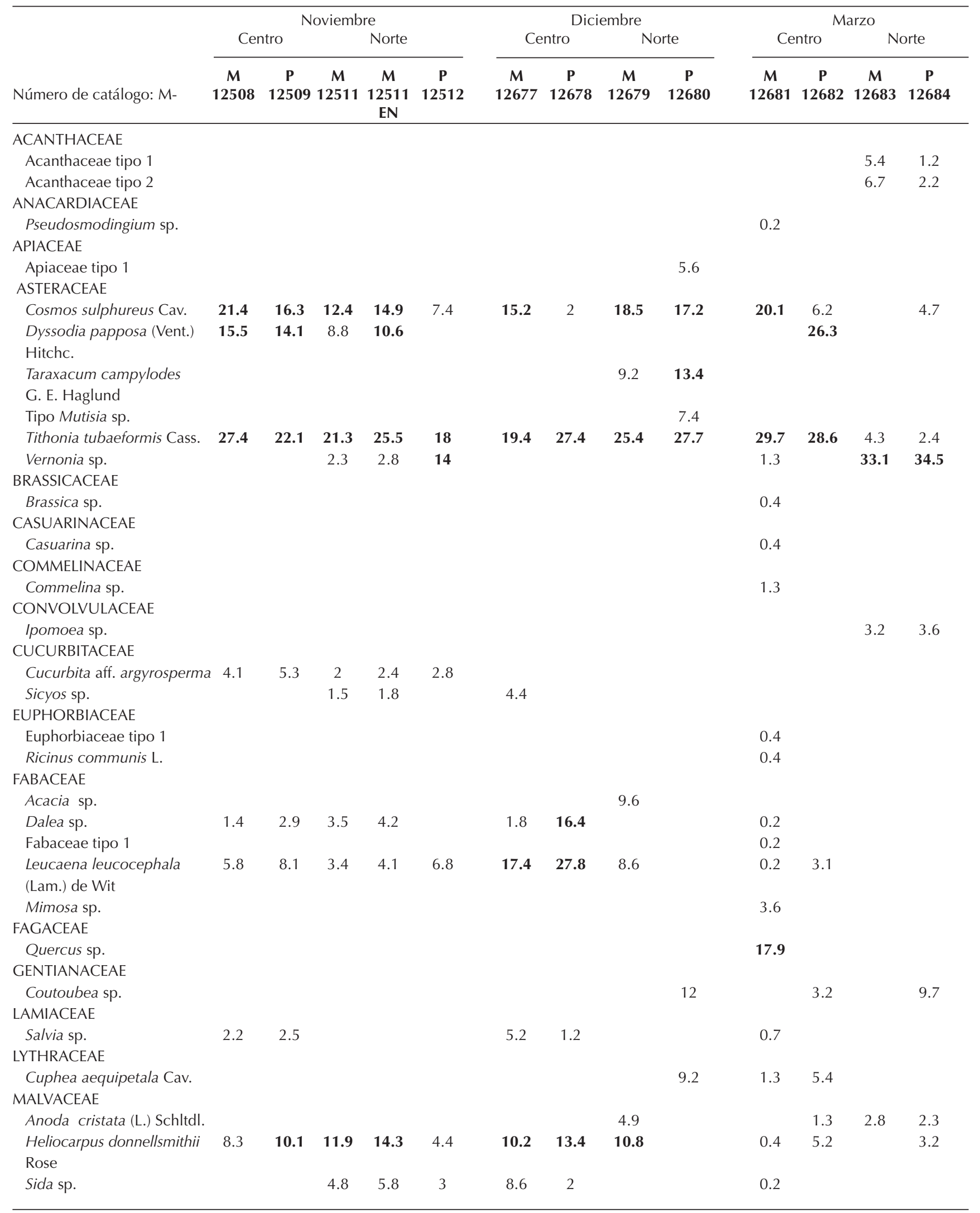


Tabla 1. Continuación

\begin{tabular}{|c|c|c|c|c|c|c|c|c|c|c|c|c|c|}
\hline \multirow[b]{3}{*}{ Número de catálogo: M- } & \multicolumn{5}{|c|}{ Noviembre } & \multicolumn{4}{|c|}{ Diciembre } & \multicolumn{4}{|c|}{ Marzo } \\
\hline & \multicolumn{2}{|c|}{ Centro } & \multicolumn{3}{|c|}{ Norte } & \multicolumn{2}{|c|}{ Centro } & \multicolumn{2}{|c|}{ Norte } & \multicolumn{2}{|c|}{ Centro } & \multicolumn{2}{|c|}{ Norte } \\
\hline & $\begin{array}{c}M \\
12508\end{array}$ & $\begin{array}{c}P \\
12509\end{array}$ & $\begin{array}{c}M \\
12511\end{array}$ & $\begin{array}{c}M \\
12511 \\
\text { EN }\end{array}$ & $\begin{array}{c}P \\
12512\end{array}$ & $\begin{array}{c}M \\
12677\end{array}$ & $\begin{array}{c}P \\
12678\end{array}$ & $\begin{array}{c}M \\
12679\end{array}$ & $\begin{array}{c}P \\
12680\end{array}$ & $\begin{array}{c}M \\
12681\end{array}$ & $\begin{array}{c}P \\
12682\end{array}$ & $\begin{array}{c}M \\
12683\end{array}$ & $\begin{array}{c}P \\
12684\end{array}$ \\
\hline \multicolumn{14}{|l|}{ MELASTOMATACEAE } \\
\hline Miconia sp. & & & & & & & & & & & & 14.5 & \\
\hline Eugenia sp. & & & & & & & & & & 8.3 & & & \\
\hline \multicolumn{14}{|l|}{ NYCTAGINACEAE } \\
\hline Pisonia sp. & & & & & & & & & & & 5.7 & 24.9 & 6.5 \\
\hline \multicolumn{14}{|l|}{ OLEACEAE } \\
\hline $\begin{array}{l}\text { Fraxinus uhdei (Wenz.) } \\
\text { Lingelsh. }\end{array}$ & 6.5 & 9.2 & 3.4 & 4.1 & & & & & & 9.4 & 7.4 & & \\
\hline \multicolumn{14}{|l|}{ POACEAE } \\
\hline Zea mays L. & & & 16.6 & & 27.4 & & & & 7.5 & & & & \\
\hline \multicolumn{14}{|l|}{ RHAMNACEAE } \\
\hline Ziziphus sp. & & & & & & & & & & 0.2 & & & \\
\hline \multicolumn{14}{|l|}{ RUTACEAE } \\
\hline Citrus sp. & & & & & & & & & & 0.2 & & & \\
\hline \multicolumn{14}{|l|}{ SAPINDACEAE } \\
\hline Dodonaea viscosa Jacq. & & & 4.5 & 5.4 & 16.2 & & & 7.4 & & & & & \\
\hline Paullinia sp. & & & & & & 12.4 & 3.7 & 5.6 & & & 2.5 & & \\
\hline \multicolumn{14}{|l|}{ SOLANACEAE } \\
\hline Datura sp. & & & & & & & & & & 0.9 & & & \\
\hline
\end{tabular}

17 taxones y 13 familias de plantas, los meses de noviembre y marzo mostraron la mayor riqueza con 10 y 12 géneros botánicos respectivamente (Tabla 1). En esta región cinco tipos polínicos fueron de importancia en muestras de miel y cargas de polen: Cosmos sulphureus, Dyssodia papposa, Tithonia tubaeformis, Leucaena leucocephala y Heliocarpus donnellsmithii, mientras que Quercus sp. y Paullinia sp. fueron exclusivas para miel y Dalea sp. sólo se presentó en muestras de cargas de polen.

Caracterización botánica. En la región Centro se analizó en el mes de noviembre una miel oligofloral de Asteraceae, mientras que para los meses de diciembre y marzo se caracterizaron mieles multiflorales destacando las familias Asteraceae, Fabaceae, Fagaceae, Malvaceae y Myrtaceae (Tabla 1; Figura 2).

El índice de diversidad (H') en las muestras de miel varió de 1.7 a 2.1 y en las cargas de polen de 1.8 a 2.1, mientras que el valor de uniformidad (J') se mantuvo en 0.9 para el caso de la miel y de 0.8-0.9 en las cargas de polen (Tabla 2). Los valores de J' indican que Apis mellifera explotó de manera uniforme los taxones importantes.

Región Norte. Un total de 24 taxones pertenecientes a 14 familias botánicas fueron registrados en las tres muestras de miel estudiadas. El mes de noviembre registró la máxima riqueza con 14 taxones, mientras que en diciembre y marzo se observaron nueve taxones. En el caso de las cargas de polen, se registraron 31 taxones de 14 familias, la mayor riqueza se observó en el mes de marzo con 11 taxones, mientras que en noviembre y diciembre se registraron nueve y ocho taxones respectivamente (Tabla 1). En la región Norte tres taxones fueron de interés en miel y cargas de polen: Cosmos sulphureus, Tithonia tubaeformis y Vernonia sp. Taxones exclusivos de miel fueron Dyssodia papposa, Heliocarpus donnellsmithii, Miconia sp. y Pisonia sp., mientras que plantas explotadas intensamente solo para la colecta de polen fueron Dodonaea viscosa, Solanum sp., Taraxacum campylodes y Zea mays (Tabla 1; Figura 2).

Caracterización botánica. La miel recolectada en el mes de diciembre fue clasificada como oligofloral de Asteraceae. Además, se caracterizaron dos mieles multiflorales, la primera miel fue colectada en noviembre y la segunda fue colectada en el mes de marzo, siendo las familias representativas Asteraceae, Malvaceae, Melastomataceae y Nyctaginaceae (Tabla 1; Figura 2).

Los valores de índice de diversidad variaron de 1.8 a 2.3 en la miel, mientras que los valores en las cargas de polen 


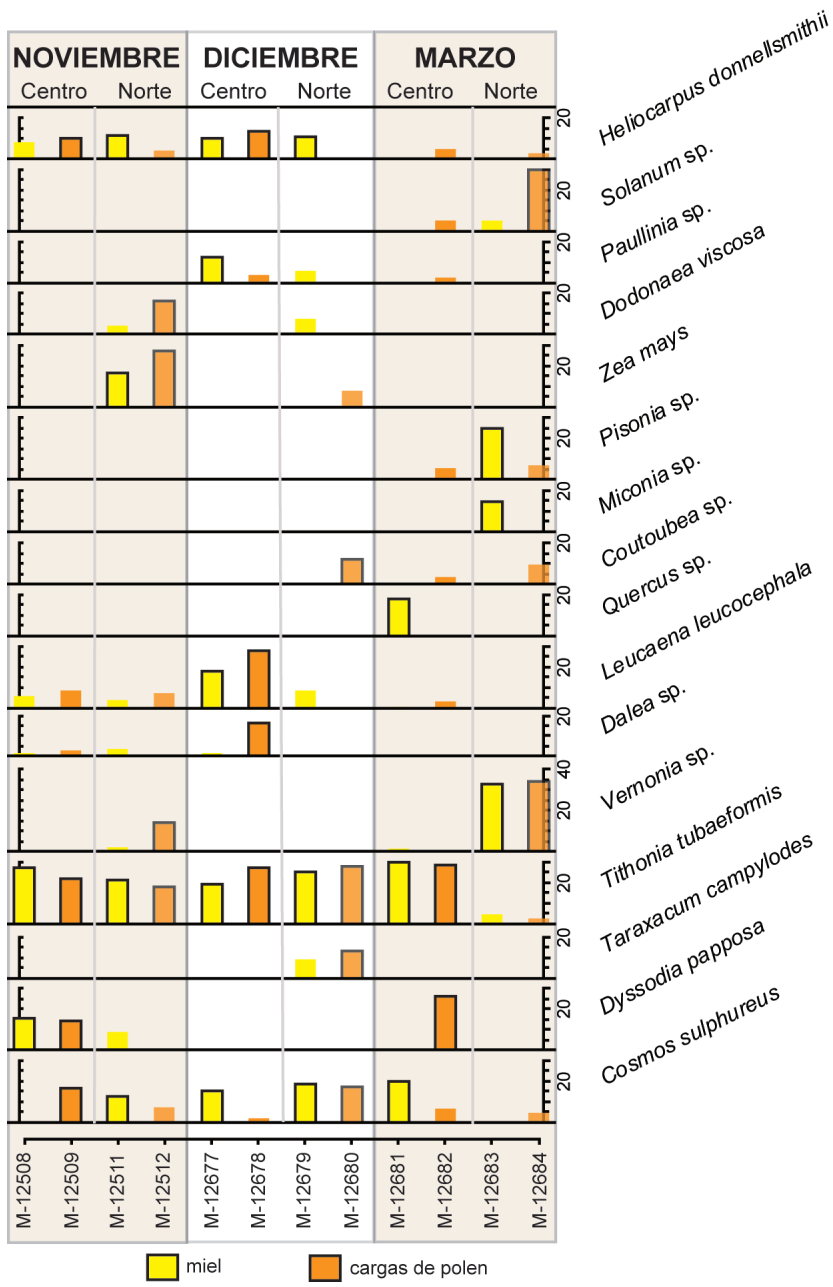

Figura 2. Recursos importantes registrados en las muestras de miel y cargas de polen de Apis mellifera en la zona Centro y Norte del estado de Guerrero. Las barras enmarcadas con negro corresponden a registros $\geq$ al $10 \%$.

analizadas fueron de 1.6 a 2. Por otro lado, los valores de uniformidad variaron de 0.8 a 1 en las muestras de miel y en las cargas de polen se registraron valores de 0.7 - 0.9 como reflejo de la uniformidad de recolecta de taxones importantes (Tabla 2).

Análisis General. En las 12 muestras analizadas, se registraron 43 taxones en total (Tabla 1; Figura 2) pertenecientes a 27 familias botánicas. Diecisiete taxones presentaron porcentajes $\geq 10 \%$, de los cuales 16 corresponden a la flora nativa: Cosmos sulphureus, Dyssodia papposa, Taraxacum campylodes, Tithonia tubaeformis y Vernonia sp. (Asteraceae), Dalea sp. y Leucaena leucocephala (Fabaceae), Quercus sp. (Fagaceae), Coutoubea sp. (Gentianaceae), Cuphea aequipetala (Lytrhaceae), Heliocarpus donnellsmithii (Malvaceae), Miconia sp. (Melastomataceae), Pisonia sp. (Nyctaginaceae), Dodonaea viscosa y Paullinia sp. (Sapindaceae) y Solanum sp. (Solanaceae). Adicionalmente, se registró la planta cultivada Zea mays (Poaceae) (Tabla 1; Figura 2).
Tabla 2. Valores de diversidad (Shanon-Weaver, $\mathrm{H}^{\prime}$ ) y de uniformidad (Pielou, $J^{\prime}$ ) de las muestras de miel y cargas de polen de Apis mellifera en la región Norte y Centro de Guerrero.

\begin{tabular}{|c|c|c|c|c|}
\hline & \multicolumn{4}{|c|}{ índice de diversidad $\mathrm{H}^{\prime}$} \\
\hline & \multicolumn{2}{|c|}{ MIEL } & \multicolumn{2}{|c|}{ POLEN } \\
\hline & centro & norte & centro & norte \\
\hline noviembre & 2 & 2.3 & 2.1 & 2 \\
\hline diciembre & 2.1 & 2.1 & 1.8 & 2 \\
\hline \multirow[t]{4}{*}{ marzo } & 1.7 & 1.8 & 2.1 & 1.6 \\
\hline & \multicolumn{4}{|c|}{ índice de uniformidad J' } \\
\hline & \multicolumn{2}{|c|}{ MIEL } & \multicolumn{2}{|c|}{ POLEN } \\
\hline & centro & norte & centro & norte \\
\hline noviembre & 0.9 & 0.9 & 0.9 & 0.9 \\
\hline diciembre & 0.9 & 0.8 & 0.9 & 0.9 \\
\hline marzo & 0.9 & 1 & 0.8 & 0.7 \\
\hline
\end{tabular}

La abeja común se desplazó preferentemente sobre el estrato herbáceo, siguiendo en importancia el estrato arbóreo y el arbustivo (Figura 3). En el estrato herbáceo fueron importantes Tithonia tubaeformis, Cosmos sulphureus, Vernonia sp., Dyssodia papposa, Zea mays, Solanum sp., Dodonaea viscosa, Dalea sp., Coutoubea sp. y Taraxacum campylodes. Además, Apis mellifera explotó el estrato arbóreo cuando se desplazó sobre Leucaena leucocephala, Heliocarpus donnellsmithii, Pisonia sp. y Quercus sp. Por último, la abeja melífera visitó intensamente Paullinia sp. y Miconia sp. del estrato arbustivo (Figura 3).

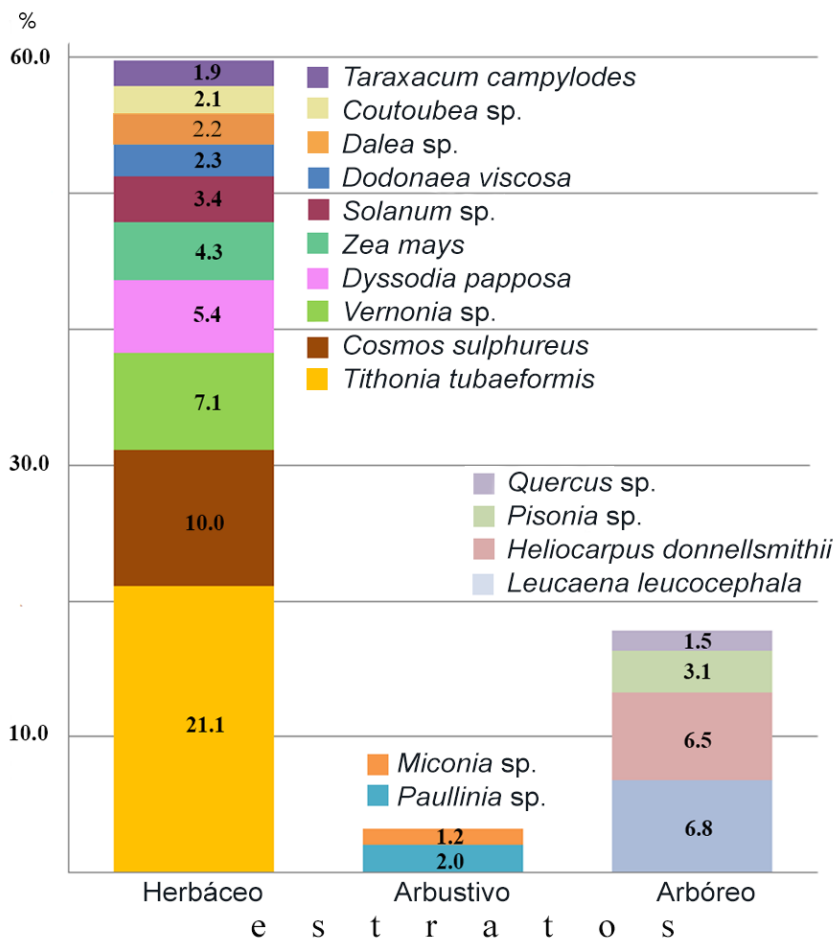

Figura 3. Estratos visitados intensamente por Apis mellifera. Porcentajes generales recalculados con base en los 6,000 granos de polen contados al azar en las 12 muestras analizadas. 
Descripciones palinológicas. En esta sección se describen exclusivamente los taxones con porcentajes $\geq 10 \%$ en las muestras analizadas, se registraron datos sobre las aberturas, características de exina con sus dimensiones, relación sexina-nexina, dimensiones del eje polar (EP); eje ecuatorial (EE); asociación, polaridad, simetría y forma (APSF), asimismo se especifica el número de catálogo de la muestra palinológica.

\section{ASTERACEAE}

Cosmos sulphureus Cav. (Figura 4A)

Polen tricolporado, endoabertura lalongada de $5.8 \mu \mathrm{m}$ (5.4$6.4 \mu \mathrm{m})$ de largo por $0.9 \mu \mathrm{m}(0.8-1.6 \mu \mathrm{m})$ de ancho, generalmente constreñida en la parte media. Área polar media. Exina tectada, columelada, supraequinada, con un patrón microrreticulado, espinas de $4.1 \mu \mathrm{m}(4-4.8 \mu \mathrm{m})$ de largo y $2.4 \mu \mathrm{m}(1.6-3.2 \mu \mathrm{m})$ en la base, distancia entre espinas de $2.2 \mu \mathrm{m}$ (1.6-2.4 $\mu \mathrm{m})$; presencia de columelas en la base de las espinas. Exina de $0.8 \mu \mathrm{m}$ sin espinas. Relación sexina-nexina entre las espinas: 2:1. APSF: Mónade, isopolar, radiosimétrica, oblato esferoidal. EP: $17.9 \mu \mathrm{m}(16-19.2 \mu \mathrm{m})$ sin espinas. EE: $17.8 \mu \mathrm{m}(16-19.2 \mu \mathrm{m})$ sin espinas. Colección Palinológica IGLUNAM: M-12679

Dyssodia papposa (Vent.) Hitchc. (Figuras 4B; 6C)

Polen tricolporado, endoabertura lalongada de $8 \mu \mathrm{m}$ de largo por $2.1 \mu \mathrm{m}$ (1.6-2.4 $\mu \mathrm{m})$ de ancho, generalmente constreñida en la parte media. Área polar media. Exina tectada, supraequinada, con un patrón microrreticulado, espinas de $4.2 \mu \mathrm{m}$ (4-4.8 $\mu \mathrm{m})$ de largo y $2.3 \mu \mathrm{m}(1.6-3.2 \mu \mathrm{m})$ en la base, distancia entre espinas de $2.6 \mu \mathrm{m}(2.4-3.2 \mu \mathrm{m})$; presencia de columelas en la base de las espinas. Exina de $1.6 \mu \mathrm{m}$ sin espinas. Relación sexina-nexina entre las espinas: 2:1. APSF: Mónade, isopolar, radiosimétrica, oblato esferoidal. EP: 20 $\mu \mathrm{m}(18.4-21.6 \mu \mathrm{m})$ sin espinas. EE: $20 \mu \mathrm{m}(17.6-21.6 \mu \mathrm{m})$ sin espinas. Colección Palinológica IGLUNAM: M-12682

Taraxacum campylodes G.E. Haglund (Figura 4G)

Polen tricolporado, endoabertura lalongada de $14.4 \mu \mathrm{m}$ (12.8-16 $\mu \mathrm{m})$ de largo por $4.6 \mu \mathrm{m}(4.5-4.8 \mu \mathrm{m})$ de ancho, generalmente constreñida en la parte media. Área polar grande. Exina tectada, equinolofada, espinas de $8.1 \mu \mathrm{m}$ (6.4-11.2 $\mu \mathrm{m})$ de largo y $3.8 \mu \mathrm{m}(3.2-4.8 \mu \mathrm{m})$ en la base, distancia entre espinas de $2.5 \mu \mathrm{m}(1.6-3.2 \mu \mathrm{m})$. Lagunas grandes de $5.3 \mu \mathrm{m}$ (4.8-6.4) $\mu \mathrm{m}$ de diámetro, muros de 0.83 $\mu \mathrm{m}(2.4-3.2 \mu \mathrm{m})$. Exina de $1.6 \mu \mathrm{m}(0.8 \mu \mathrm{m})$ sin espinas. Relación sexina-nexina entre las espinas: 3:1. APSF: Mónade, isopolar, radiosimétrica, oblato esferoidal. EP: $31 \mu \mathrm{m}$ (27.2-33.6 $\mu \mathrm{m}) \sin$ espinas. EE: $31 \mu \mathrm{m}(25.6-33.6 \mu \mathrm{m}) \sin$ espinas. Colección Palinológica IGLUNAM: M-12683

Tithonia tubaeformis Cass. (Figuras 4C; 6A, B)

Polen tricolporado, endoabertura lalongada de $10.5 \mu \mathrm{m}$ (9.6$15.2 \mu \mathrm{m})$ de largo por $1.3 \mu \mathrm{m}(0.8-1.6 \mu \mathrm{m})$ de ancho, generalmente constreñida en la parte media. Área polar media. Exina tectada, supraequinada, con un patrón microreticulado, espinas de $6.6 \mu \mathrm{m}(4.8-8 \mu \mathrm{m})$ de largo y $3.3 \mu \mathrm{m}(2.4-5.6$ $\mu \mathrm{m})$ en la base, distancia entre espinas de $2.6 \mu \mathrm{m}$ (2.4-3.2 $\mu \mathrm{m})$; presencia de columelas en la base de las espinas. Exina $1.7 \mu \mathrm{m}(1.6-2.4 \mu \mathrm{m})$ sin espinas. Relación sexina-nexina: 1:1. APSF: Mónade, isopolar, radiosimétrica, oblato esferoidal. EP: $27.1 \mu \mathrm{m}(24-33.6 \mu \mathrm{m})$ sin espinas. EE: $27 \mu \mathrm{m}$ (22.4-35.2 $\mu \mathrm{m}$ ) sin espinas. Colección Palinológica IGLUNAM: M-12682

Vernonia sp. (Figuras 4E, F; 6D, E)

Polen tricolporado, endoabertura lalongada de 11-13 $\mu \mathrm{m}$ de largo, constreñida en la parte media, con dos micras de ancho a ambos lados de dicha constricción. Área polar pequeña. Exina tectada, equinolofada, con un patrón microrreticulado, espinas de $5.5 \mu \mathrm{m}(5-7 \mu \mathrm{m})$ de largo y $2.2 \mu \mathrm{m}(2-3$ $\mu \mathrm{m})$ en la base, distancia entre espinas de $8 \mu \mathrm{m}(6-10 \mu \mathrm{m})$. Lagunas chicas de 4-5 $\mu \mathrm{m}$ de ancho y lagunas grandes de 8 a $13 \mu \mathrm{m}$ de diámetro, muros de $4-5 \mu \mathrm{m}$ y de $7-10 \mu \mathrm{m}$ en las intersecciones. Exina de 5-7 $\mu \mathrm{m}$ sin espinas. Relación sexina-nexina entre las espinas: 6:1. APSF: Mónade, isopolar, radiosimétrica, oblato esferoidal. EP: $38.7 \mu \mathrm{m}(34-42 \mu \mathrm{m})$ sin espinas. EE: $40.8 \mu \mathrm{m}(37-46 \mu \mathrm{m})$ sin espinas. Colección Palinológica IGLUNAM: M-12512, M-12679, M-1268

\section{FAGACEAE}

Quercus sp. (Figuras 4L, M; 7E, F)

Polen tricolpado, tricolporoidado. Área polar media. Exina tectada, columelada, supraverrugada. Exina de $2 \mu \mathrm{m}$ de grosor. Relación sexina-nexina: 1:1. APSF: Mónade, isopolar, radiosimétrica, prolato esferoidal a subprolato. EP: $34 \mu \mathrm{m}$ (30-40 $\mu \mathrm{m})$. EE: $30.8 \mu \mathrm{m}(25-35 \mu \mathrm{m})$. Colección Palinológica IGLUNAM: M-12681

\section{GENTIANACEAE}

\section{Coutoubea sp. (Figuras 4K, N)}

Polen tricolporado, endoabertura lalongada de $4.9 \mu \mathrm{m}$ (4.8$5.6 \mu \mathrm{m})$ de largo por $4.1 \mu \mathrm{m}(3.2-4.8 \mu \mathrm{m})$ de ancho. Área polar pequeña. Exina semitectada, ornamentación reticula$\mathrm{da}$, heterobrocada, muros menores a $1 \mu \mathrm{m}$ y luminas de 2.8 $\mu \mathrm{m}(2-4 \mu \mathrm{m})$ con microverrugas en las lúminas. Exina de $2.7 \mu \mathrm{m}$ (1.6-3.2) $\mu \mathrm{m}$. Relación sexina-nexina: 3:1. APSF: Mónade, isopolar, radiosimétrica, oblato esferoidal. EP: 64 $\mu \mathrm{m}(52-74.4 \mu \mathrm{m})$. EE: $60.4 \mu \mathrm{m}(50.4-70.4 \mu \mathrm{m})$. Colección Palinológica IGLUNAM: 12680

\section{FABACEAE}

Dalea sp. (Figuras 4D, H)

Polen tricolpado, área polar pequeña. Exina subtectada columelada, microrreticulada, homobrocada. Exina de 1-2 $\mu \mathrm{m}$ de grosor, lúminas menores de $1 \mu \mathrm{m}$. Relación sexina-nexina: 1:1. APSF: Mónade, isopolar, radiosimétrica, perprolato, ocasionalmente prolato. EP: $43.8 \mu \mathrm{m}(38-53 \mu \mathrm{m})$. EE: $19.4 \mu \mathrm{m}(15-23 \mu \mathrm{m})$. Colección Palinológica IGLUNAM: M-12677, M-12682

Leucaena leucocephala (Lam.) de Wit (Figuras 4P, Q; $7 \mathrm{~A}, \mathrm{~B})$ 

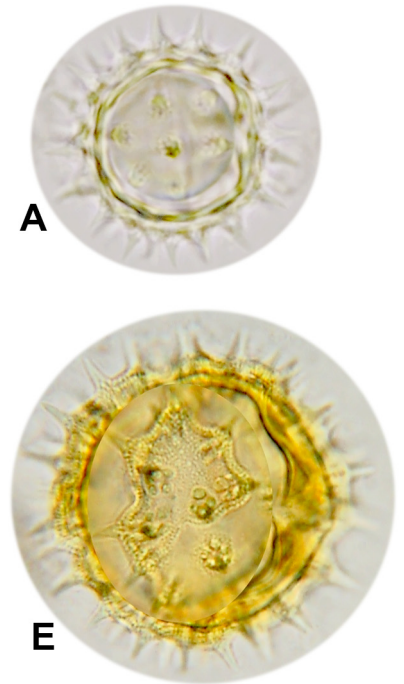

B
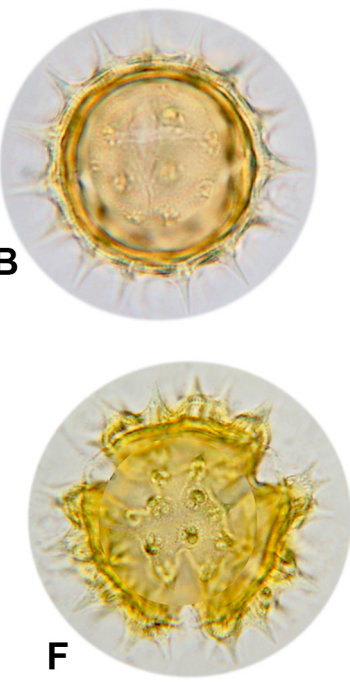

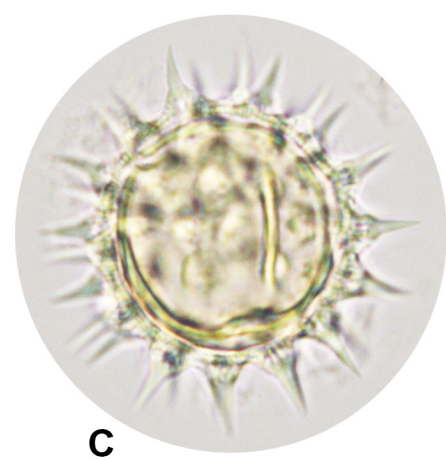

C

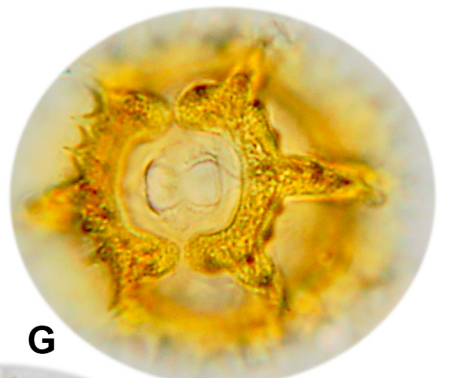

$10 \mu \mathrm{m}$
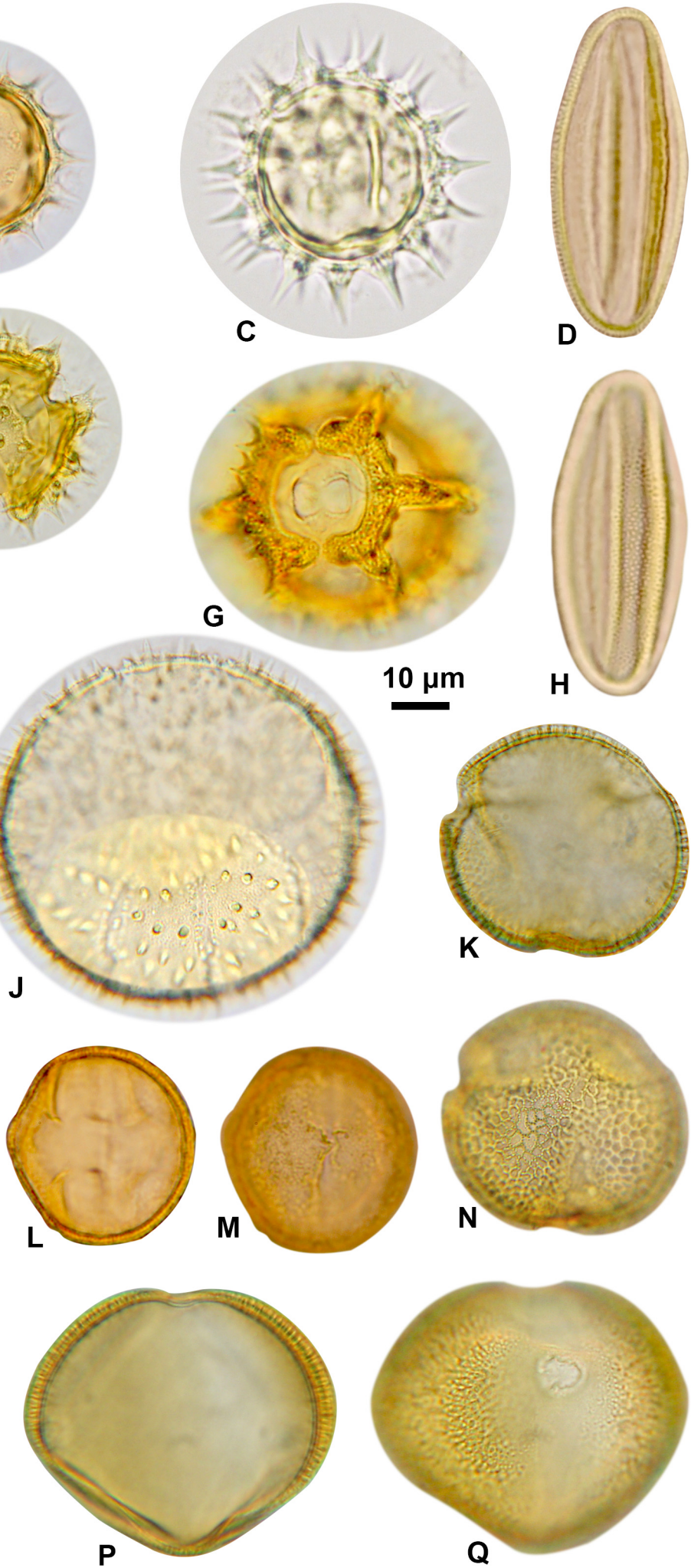

Figura 4. Micrografías en microscopio óptico del polen recuperado en las muestras de miel y cargas de polen de Apis mellifera en el estado de Guerrero. Asteraceae: A. Cosmos sulphureus. B. Dyssodia papposa. G. Taraxacum campylodes. C. Tithonia tubaeformis. E, F. Vernonia sp. Cucurbitaceae: J. Cucurbita aff. argyrosperma. Fabaceae: D, H. Dalea sp. P, Q. Leucaena leucocephala. Fagaceae: L, M. Quercus sp. Gentianaceae: K, N. Coutoubea sp. Malvaceae: I, O. Anoda cristata. La escala representa $10 \mu \mathrm{m}$. 

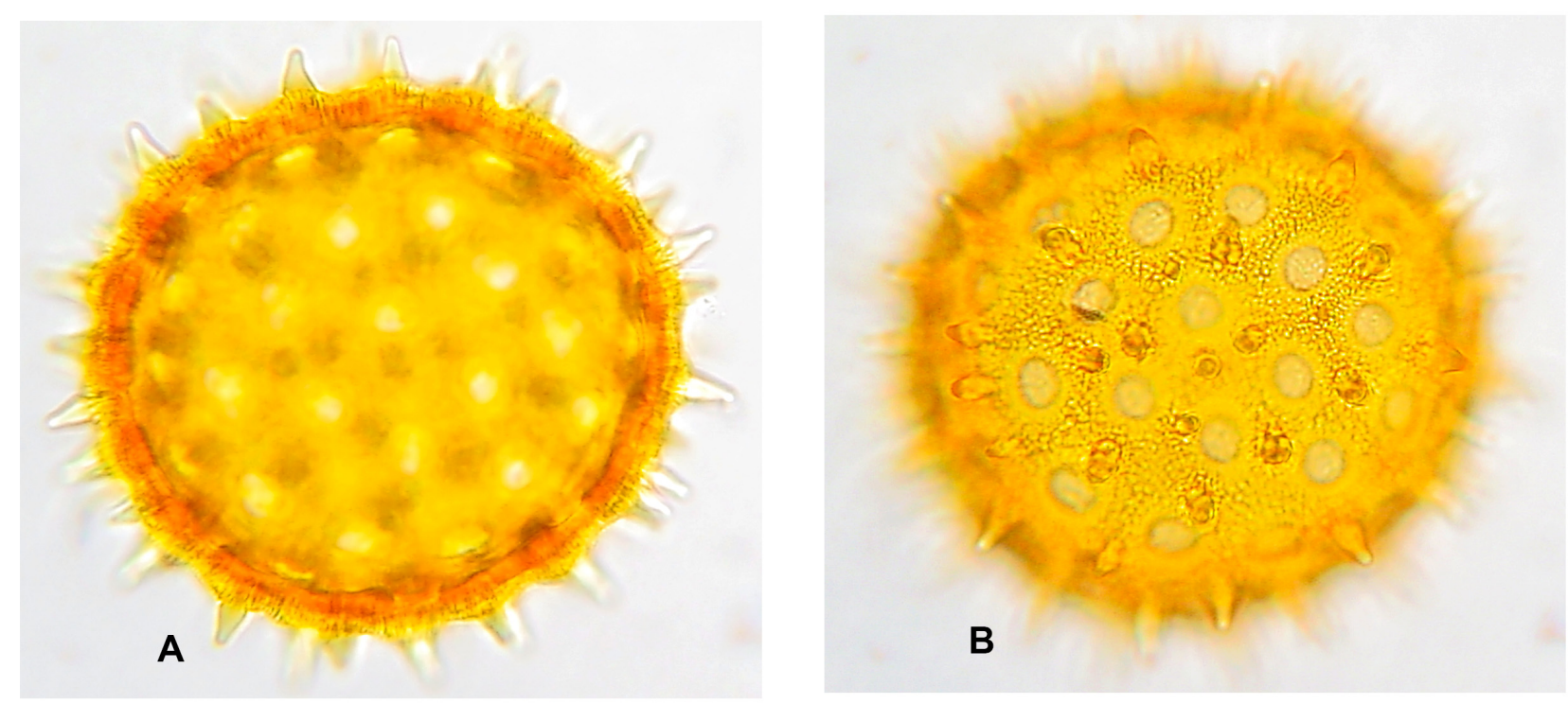
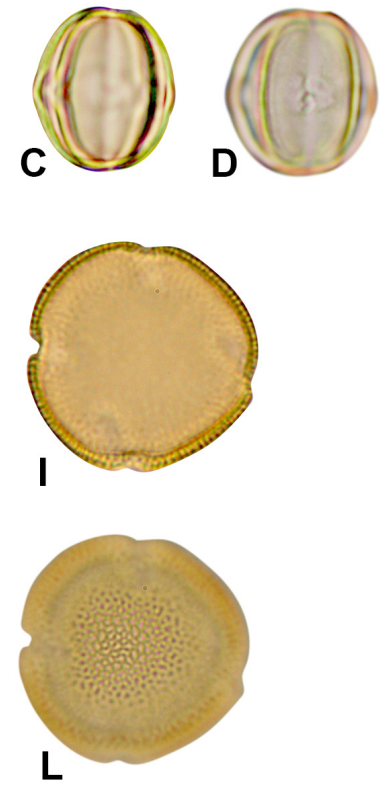

$\mathbf{L}$
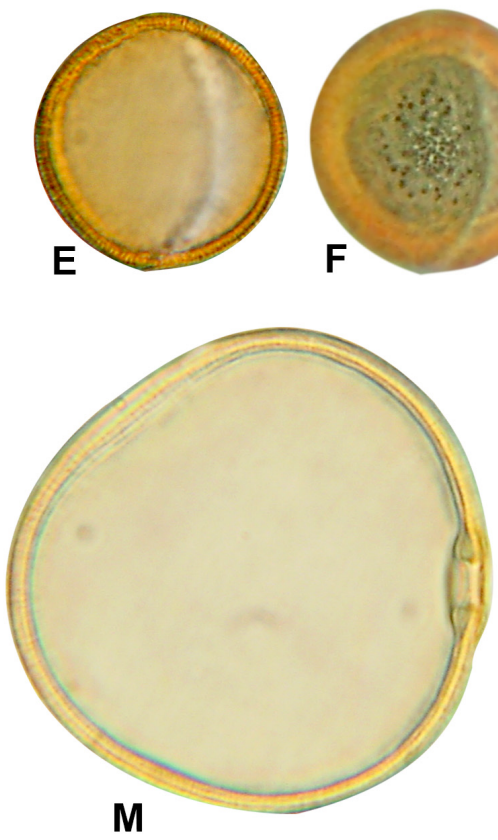
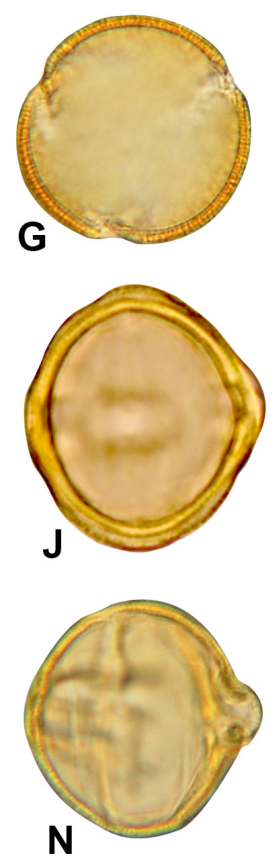

$\mathrm{H}$
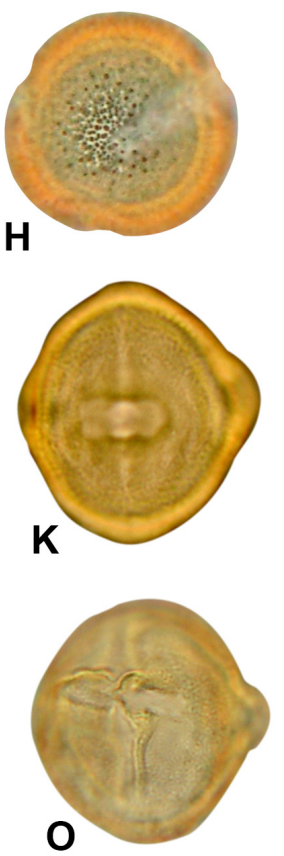
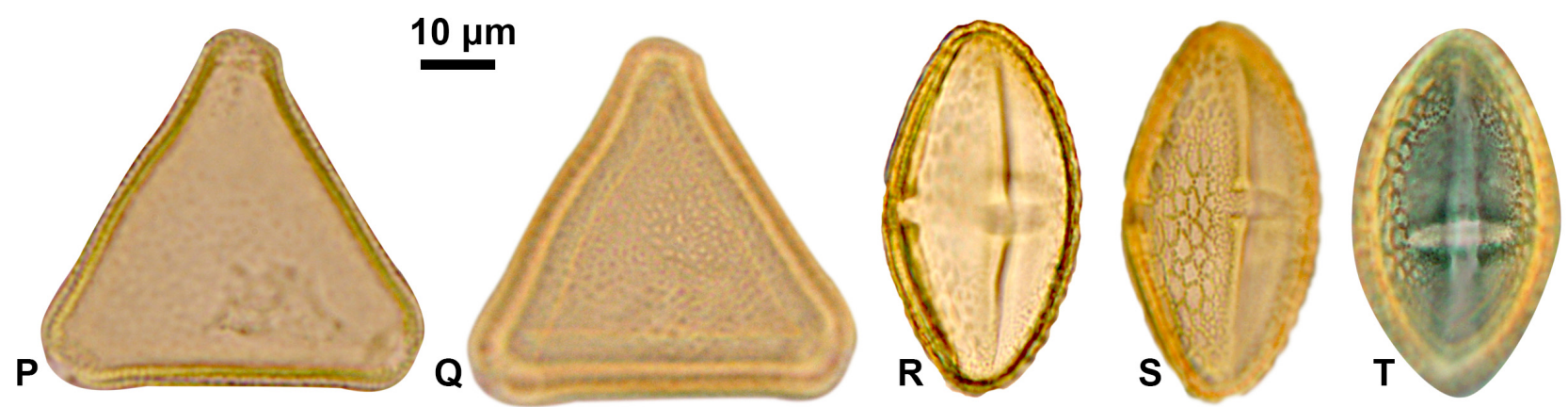

Figura 5. Micrografías en microscopio óptico del polen recuperado en las muestras de miel y cargas de polen de Apis mellifera en el estado de Guerrero. Malvaceae: A, B. Sida sp. Melastomataceae: C, D. Miconia sp. Nyctaginaceae: E-H. Pisonia sp. Oleaceae: I, L. Fraxinus uhdei. Poaceae: M. Zea mays. Sapindaceae: J, K. Dodonaea viscosa. P, Q. Paullinia sp. Solanaceae: N, O. Solanum sp. Malvaceae: R-T. Heliocarpus donnellsmithii. La escala representa $10 \mu \mathrm{m}$. 
Polen tricolporado, endoabertura circular 5-7 $\mu \mathrm{m}$, área polar pequeña. Exina tectada, columelada, microfoveolada, con patrón microrreticulado. Exina de 2-3 $\mu \mathrm{m}$ de grosor. Relación sexina-nexina: 2:1. APSF: Mónade, isopolar, radiosimétrica, oblato esferoidal. EP: $50 \mu \mathrm{m}(43-56 \mu \mathrm{m})$. EE: 52.1 $\mu \mathrm{m}(45-57 \mu \mathrm{m})$. Colección Palinológica IGLUNAM: M-12511, M-12512, M-12677, M-12678, M-12682

\section{MALVACEAE}

Heliocarpus donnellsmithii Rose (Figuras 5R, S, T; 7C, D) Polen tricolporado, endoabertura lalongada de $12 \mu \mathrm{m}$ (10-15 $\mu \mathrm{m})$ de largo y $2.7 \mu \mathrm{m}(2-4 \mu \mathrm{m})$ de ancho. Área polar media. Exina subtectada, columelada, ornamentación reticulada, heterobrocada. Luminas grandes de $4.5 \mu \mathrm{m}(4-6 \mu \mathrm{m})$ y luminas pequeñas de $3 \mu \mathrm{m}$, los muros son simplicolumelados de 1-1.6 $\mu \mathrm{m}$ de ancho. Exina de $2.1 \mu \mathrm{m}(2-3 \mu \mathrm{m})$ de grosor. Relación sexina-nexina: 2:1. APSF: Mónade, isopolar, radiosimétrica, prolato. EP: $46 \mu \mathrm{m}(39-55 \mu \mathrm{m})$. EE: $28.6 \mu \mathrm{m}$ (26-31 $\mu \mathrm{m})$. Colección Palinológica IGLUNAM: M-1208, M-12511, M-12512, M-12677, M-12679.

\section{MELASTOMATACEAE}

Miconia sp. (Figuras 5C, D)

Polen heterocolpado con 3 colpos y tres aberturas colporadas intercaladas entre las aberturas simples. Endoabertura lalongada constreñida en la parte media, de $4 \mu \mathrm{m}$ de largo y $2 \mu \mathrm{m}$ de ancho a ambos lados de la constricción. Exina tectada, columelas imperceptibles, psilada. Exina de 1-1.5 $\mu \mathrm{m}$ de grosor. Relación sexina-nexina: 1:1. APSF: Mónade, isopolar, radiosimétrica, subprolato. EP: $19.5 \mu \mathrm{m}$ (17$21 \mu \mathrm{m})$. EE: $15.3 \mu \mathrm{m}(13-17 \mu \mathrm{m})$. Colección Palinológica IGLUNAM: M-12683, M-12684

\section{NYCTAGINACEAE}

Pisonia sp. (Figuras 5E, F, G, H)

Polen tricolpado. Área polar media. Exina tectada, columelada, supramicroequinada con un patrón microrreticulado. Exina de 2-3 $\mu \mathrm{m}$ de grosor. Relación sexina-nexina: 1:1. EP: $34.6 \mu \mathrm{m}(30-38 \mu \mathrm{m})$. EE: $31.8 \mu \mathrm{m}(27-35 \mu \mathrm{m})$. APSF: Mónade, isopolar, radiosimétrica, prolato esferoidal a subprolato. Colección Palinológica IGLUNAM: M-12683

\section{POACEAE}

Zea mays L. (Figuras 5M; 6F, G)
Polen monoporado con anulus, poro de $1.4 \mu \mathrm{m}(2.4-3.2 \mu \mathrm{m})$ de diámetro. Exina tectada, supraverrugada con patrón areolado $1.7 \mu \mathrm{m}$ (1.6-2.4 $\mu \mathrm{m})$. Relación sexina-nexina: 1:1. EP: $46 \mu \mathrm{m}(40.0-56 \mu \mathrm{m})$. EE: $41.6 \mu \mathrm{m}(35.2-48 \mu \mathrm{m})$. APSF: Mónade, heteropolar, radiosimétrico, de oblato esferoidal a subprolato. Colección Palinológica IGLUNAM: M-12511

\section{SAPINDACEAE}

Dodonaea viscosa Jacq. (Figura 5J, K)

Polen tricolporado, ectoabertura con margo. Endoabertura lalongada con costae convergentes y márgenes meridionales cerrados de $7.3 \mu \mathrm{m}(5-10 \mu \mathrm{m})$ de largo y $3.9 \mu \mathrm{m}(3-5 \mu \mathrm{m})$ de ancho. Costae de 0.8-1.5 $\mu \mathrm{m}$ de grosor. Área polar media. Exina tectada, columelada, ornamentación supamicroverrugada, formando un retículo negativo, con endofisuras en la nexina. Exina de $2.8 \mu \mathrm{m}(2-3 \mu \mathrm{m})$ de grosor. Relación sexina-nexina: 2:1. APSF: Mónade, isopolar, radiosimétrica, prolato esferoidal, rara vez subprolato. EP: $31.8 \mu \mathrm{m}$ (30$35 \mu \mathrm{m})$. EE: $29.8 \mu \mathrm{m}(26-33 \mu \mathrm{m})$. Colección Palinológica IGLUNAM: M-12511, M-12512, M-12679

Paullinia sp. (Figura 5P, Q)

Polen triporado, poros de $0.8 \mu \mathrm{m}$ de diámetro. Exina subtectada, columelada, reticulada y heterobrocada. Exina de 1.1 $\mu \mathrm{m}(1-1.5 \mu \mathrm{m})$ de grosor, luminas de 1-1.5 $\mu \mathrm{m}$ de diámetro. Relación sexina-nexina: 1:1. APSF: Mónade, isopolar, radiosimétrica, oblato. EE: $26.7 \mu \mathrm{m}(24-31 \mu \mathrm{m})$. Colección Palinológica IGLUNAM: M-12677

\section{Discusión}

Recursos de interés melífero y polinífero. La familia Asteraceae es una de las más abundantes y diversas en México con 392 géneros y alrededor de 3,005 especies (Balleza y Villaseñor, 2002). Espina y Ordetx (1983) destacan que numerosas especies de Asteraceae son fuente de néctar y polen para Apis. De acuerdo a las colectas botánicas realizadas en las dos regiones de estudio, la familia Asteraceae registró mayor número de especies para la región Centro. Además, por presentarse como elementos importantes en las muestras de miel y cargas de polen de este trabajo se le consideró como una familia nectaro-polinífera. Trabajos melisopalinológicos realizados en el Centro de México: en los estados de Morelos, Zacatecas y Distrito Federal (Piedras-Gutiérrez y Quiroz- García, 2007; Quiroz-García et al., 2011; Acosta-

Tabla 3. [HT1]Valores de diversidad (Shanon-Weaver, $\mathrm{H}^{\prime}$ ) y uniformidad (Pielou, J') de las muestras de miel y cargas de polen en los estados de Guerrero (presente estudio), Oaxaca (Ramírez-Arriaga et al., 2011), Puebla (Ramírez-Arriaga y Martínez-Hernández, 2007) y Tabasco (Castellanos-Potenciano, 2010; Castellanos-Potenciano et al., 2012).

\begin{tabular}{|c|c|c|c|c|c|c|c|c|c|c|c|c|c|}
\hline & \multicolumn{6}{|c|}{ Guerrero } & \multicolumn{3}{|c|}{ Oaxaca } & \multirow{3}{*}{$\begin{array}{c}\text { Puebla } \\
\text { Miel }\end{array}$} & \multicolumn{3}{|c|}{ Tabasco } \\
\hline & \multicolumn{2}{|c|}{ Max } & \multicolumn{2}{|c|}{ Min } & \multicolumn{2}{|c|}{ Prom } & \multirow{2}{*}{$\begin{array}{l}\text { Max } \\
\text { Miel }\end{array}$} & \multirow{2}{*}{$\begin{array}{l}\text { Min } \\
\text { Miel }\end{array}$} & \multirow[b]{2}{*}{ Prom } & & Max & Min & \\
\hline & Miel & Polen & Miel & Polen & Miel & Polen & & & & & Miel & Miel & Prom \\
\hline $\mathrm{H}^{\prime}$ & 2.33 & 2.13 & 1.66 & 1.57 & 2 & 1.91 & 2.7 & 0.3 & 1.49 & 1.74 & 2.6 & 0.7 & 1.22 \\
\hline$J^{\prime}$ & 0.99 & 0.94 & 0.81 & 0.65 & 0.89 & 0.85 & 0.7 & 0.1 & 0.45 & 0.66 & 0.8 & 0.3 & 0.61 \\
\hline
\end{tabular}




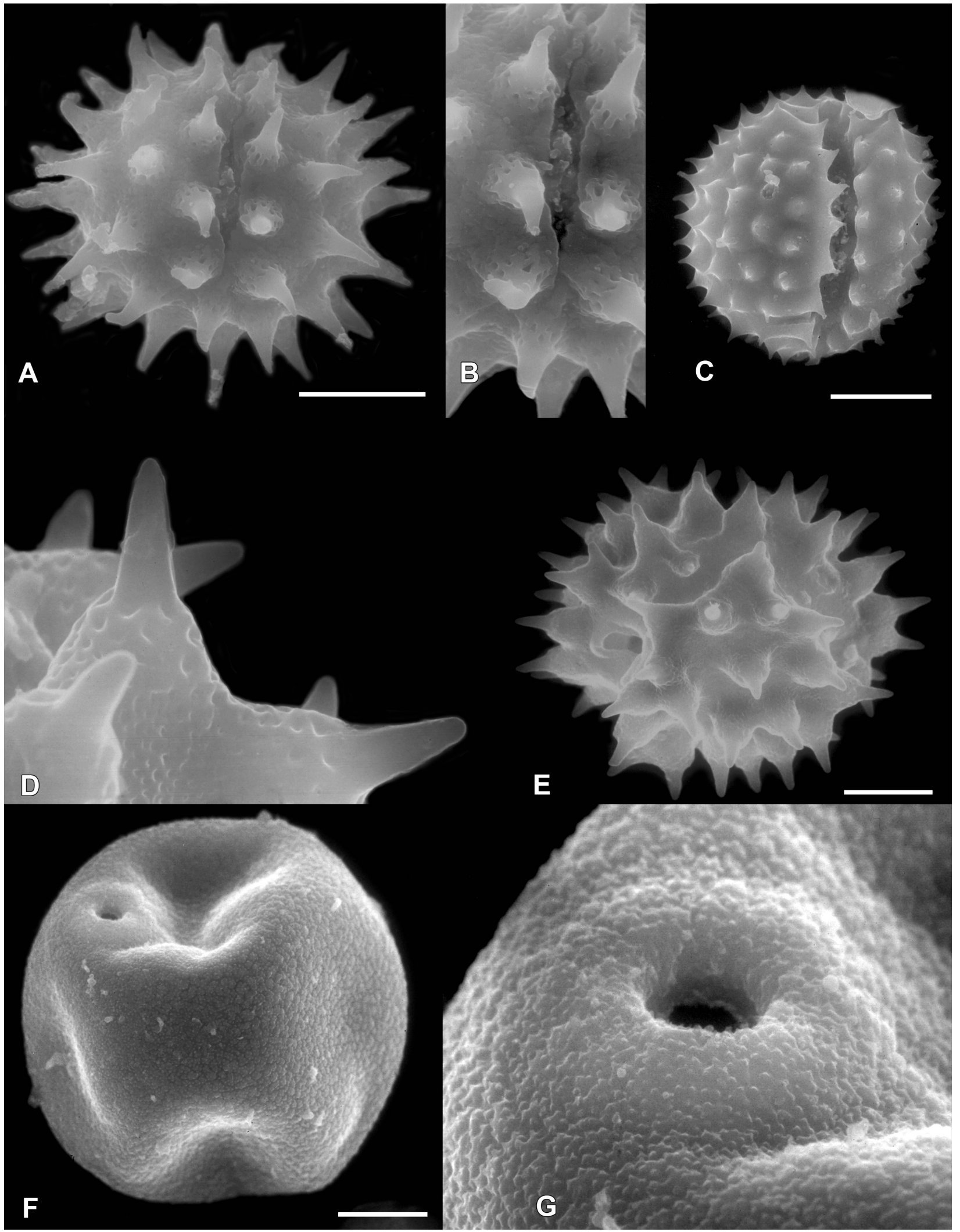

Figura 6. Micrografías en microscopio electrónico de barrido del polen recuperado en las muestras de miel y cargas de polen de Apis mellifera en el estado de Guerrero. Asteraceae: A, B. Tithonia tubaeformis. C. Dyssodia papposa. D, E. Vernonia sp. Poaceae: F, G. Zea mays. La escala representa $10 \mu \mathrm{m}$. 


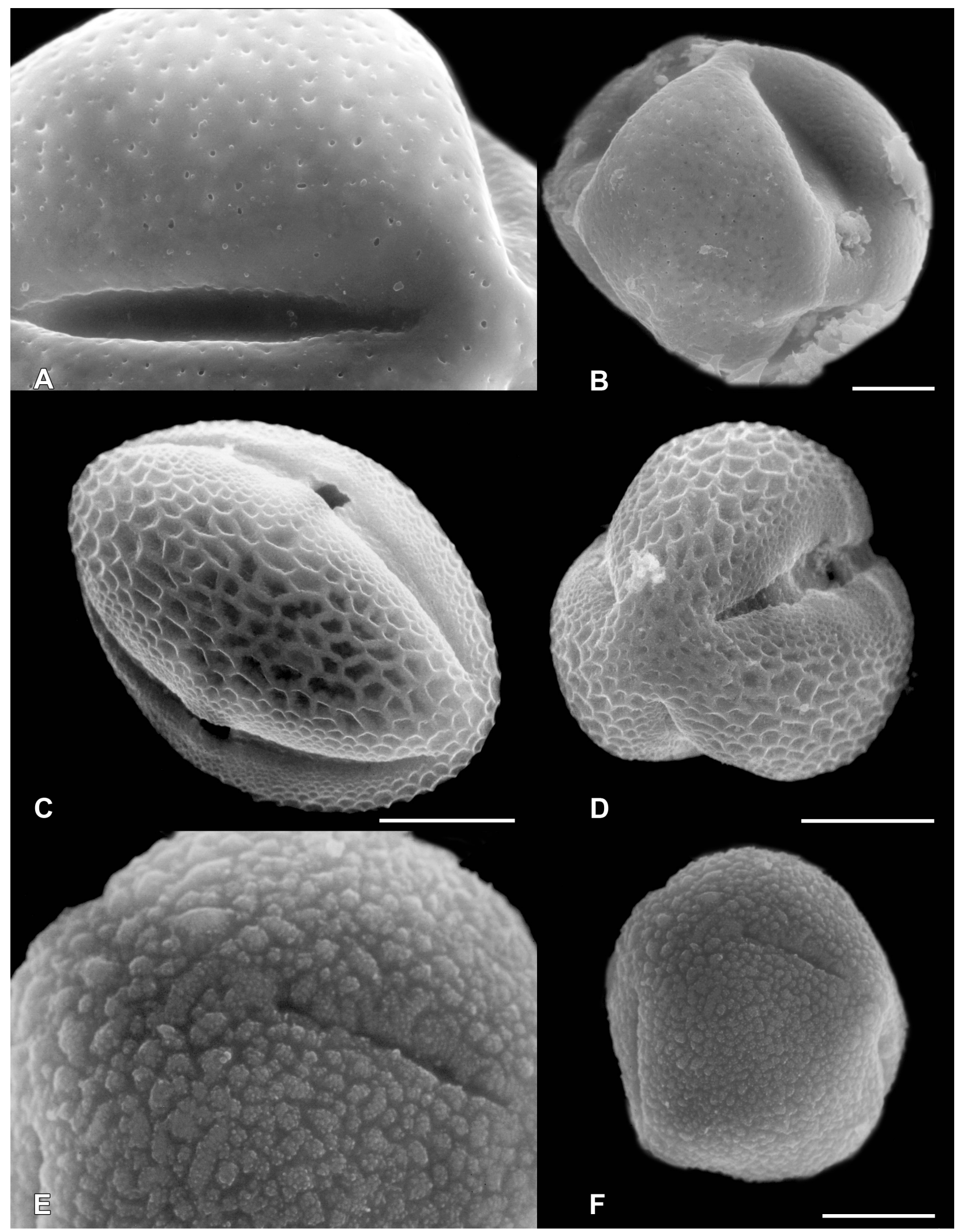

Figura 7. Micrografías en microscopio electrónico de barrido del polen recuperado en las muestras de miel y cargas de polen de Apis mellifera en el estado de Guerrero. Fabaceae: A, B. Leucaena leucocephala. Malvaceae: C, D. Heliocarpus donnellsmithii. Fagaceae: E, F. Quercus sp. La escala representa $10 \mu \mathrm{m}$. 
Castellanos et al., 2011); en el Golfo de México: en el estado de Tabasco (Castellanos-Potenciano et al., 2012), y en la región Pacífico: en el estado de Oaxaca (Ramírez- Arriaga et al., 2011), las asteráceas han sido reconocidas como de interés nectarífero y nectaro-polinífero.

La familia Fabaceae es también considerada una de las más importantes para México con 139 géneros y 1,850 especies, siendo Dalea uno de los géneros más diversos con 113 especies (Olvera-Luna et al., 2012). Esta familia registró dos especies de importancia, Leucaena leucocephala en miel y polen; así como Dalea sp. en cargas de polen. En el estudio realizado por Villanueva (2002) en cargas de polen de Apis mellifera, reportó a L. leucocephala como una planta de interés polinífero en el estado de Yucatán. Por medio de observaciones de campo, dicha especie es considerada de importancia nectarífera y polinífera en Yucatán (Villegas-Durán et al., 1998) y Guerrero (Villegas-Durán et al., 2002). Por otro lado, Villegas-Durán et al. (2003) cita varias especies del género Dalea como importantes para la producción apícola para el estado de Veracruz, con floraciones de marzo a mayo. En este estudio melisopalinológico se reporta por primera vez este género como importante en muestras de miel.

México es considerado uno de los centros de diversificación de encinos, con 161 especies de Quercus (Fagaceae), $68 \%$ de ellas son endémicas (Valencia, 2004). Los encinos son especies consideradas como poliníferas con base en estudios de campo (Espina y Ordetx 1983), sin embargo, en zonas con buen aporte pluvial, se ha observado que los árboles producen exudados llamados "mieladas" y/o "miel de bosque" que las abejas recolectan para la elaboración de miel (Ortiz, 1990; Sancho et al., 1991). En México, Quercus se ha reportado en estudios palinológicos en mieles de Zacatecas (Acosta-Castellanos et al., 2011), Oaxaca (Ramírez-Arriaga et al., 2011) y del estado de Tabasco (Castellanos-Potenciano, 2010; Castellanos-Potenciano et al., 2012). Debido a que en el presente estudio Quercus se registró como importante en una muestra de miel del mes de marzo, recolectada en la región Centro de Guerrero, se considera que dicha miel se formó a partir de una mezcla de miel de néctar de flores y mieladas de encino.

La Gentianaceae Coutoubea sp. se presentó en muestras de polen, cabe destacar que dicho género no ha sido citado previamente en trabajos melisopalinológicas ni en observaciones de campo, por lo que este es el primer reporte.

Con relación a la familia Melastomataceae, el género $\mathrm{Mi}$ conia sp., registrado como nectarífero para Guerrero, también es considerado de importancia melífera en los estados de Chiapas (Martínez-Hernández et al., 1993) y Puebla (Ramírez-Arriaga y Martínez-Hernández, 2007). Además, Ramírez-Arriaga et al. (2011) reportan M. argentea en mieles del estado de Oaxaca. Por último, la familia Melastomataceae ha sido también citada en mieles de Tabasco (Castellanos-Potenciano et al., 2012).

Pisonia sp. (Nyctaginaceae) tuvo incidencia en muestras de miel. Villegas-Durán et al. (2003) registraron este género de importancia nectarífera en la flora apícola para el estado de Veracruz con floración de febrero a abril y en la península de Yucatán. Ramírez-Arriaga et al. (2011), citaron a Pisonia sp. y Pisonia aculeata como recursos secundarios para Apis en mieles de Oaxaca.

La familia Poaceae es muy abundante y diversa en México contando con alrededor de 197 géneros y 1120 especies (Dávila y Sánchez-Ken, 1994). Esta familia estuvo representada por Zea mays en muestras de miel, por lo que se realizó un recalculo con base en especies exclusivamente nectaríferas y también se registró en cargas de polen. Villanueva (1984) menciona que dicha especie generalmente es ignorada por las abejas, sin embargo, cuando no disponen de otros recursos florales suelen visitarla, por la gran cantidad de polen producido. Piedras- Gutiérrez y Quiroz-García (2007), mencionan que el polen de Zea mays, generalmente es anemófilo y cuando se presenta en muestras de miel es de forma incidental, sin embargo, la gran producción de polen resulta ser una fuente importante de proteína para las abejas, principalmente cuando los apiarios se ubican en las proximidades de las zonas de cultivo del maíz. De igual manera, Quiroz-García y ArreguínSánchez (2008) ratifican que aun cuando Z. mays se observó en miel, es una planta exclusivamente polinífera.

La familia Sapindaceae estuvo representada por el género Dodonaea viscosa en una muestra de polen. El polen de esta especie se encontró en mieles de Oaxaca (Ramírez-Arriaga et al., 2011). Otra sapindácea registrada en las muestras de miel de Guerrero fue Paullinia sp., la cual ya ha sido reportada en muestras de miel del estado de Morelos (Quiroz-García y Arreguín-Sánchez, 2008).

Para la familia Solanaceae, el género Solanum sp. fue importante estar presente en una muestra de cargas de polen en la región Norte (Cuadro 1). Las especies $S$. rostratum y S. mammosum son plantas de interés apícola en los estados de Michoacán, Campeche y Yucatán (Villegas-Durán et al., 1998; Pacheco-Palomo, 2011).

Heliocarpus donnellsmithii (Malvaceae) fue importante en cinco muestras tanto de miel como de cargas de polen en ambas regiones de Guerrero. En investigaciones melisopalinológicas se tiene bien documentada esta especie en mieles de Oaxaca, Puebla, Morelos (Ramírez-Arriaga y Martínez-Hernández, 2007; Quiroz-García y Arreguín-Sánchez, 2008; Ramírez-Arriaga et al., 2011) y en cargas de polen en Yucatán (Villanueva, 2002); otras especies del género Heliocarpus se encuentran reportadas en mieles de Tabasco y Zacatecas (Acosta-Castellanos et al., 2011; CastellanosPotenciano et al., 2012).

Por último, es importante destacar que aquellas especies presentes en las muestras con registros menores al $10 \%$ son considerados como recursos alternativos para Apis mellifera (Ramalho et al., 1985; Ramírez-Arriaga et al., 2011).

Aspectos Ecológicos: En general, Apis mellifera presentó un 
comportamiento poliléctico, sin embargo, llegó a ser más selectiva, es decir con preferencias oligolécticas, cuando se desplazó preferentemente sobre las especies de Asteraceae. Respecto a la uniformidad de recolecta de los recursos, éste puede ser considerado homogéneo con base en los valores del índice de Pielou.

En la Tabla 3 se pueden comparar los valores de H' y J' en cuatro estados de la República Mexicana, incluyendo el presente estudio. Los índices de diversidad máximos en mieles se tienen registrados para las zonas tropicales como son Oaxaca con 2.7 (Ramírez-Arriaga et al., 2011) y Tabasco con 2.6 (Castellanos, 2010; Castellanos et al., 2012); le sigue en importancia el estado de Guerrero (2.3); mientras que las mieles menos diversas son las del estado de Puebla con 1.7 (Ramírez-Arriaga y Martínez-Hernández, 2007). Es importante destacar que los estados con más altos índices de diversidad en los análisis melisopalinológicos (Oaxaca, Tabasco y Guerrero), coinciden también con una gran riqueza florística, contrastando con el estados de Puebla con menor diversidad botánica (Villaseñor, 2003).

Con respecto a la conducta de recolecta, se registró conducta heterogénea en los estados de Oaxaca (Ramírez-Arriaga et al., 2011), Puebla (Ramírez-Arriaga y Martínez-Hernández, 2007) y Tabasco (Castellanos-Potenciano, 2010; Castellanos-Potenciano et al., 2012). Mientras que, comportamientos de recolecta homogénea se registraron en Guerrero y ocasionalmente en los estados de Oaxaca y Tabasco.

\section{Conclusiones}

En México existe una gran tradición apícola, como queda de manifiesto por ser un gran productor de miel (Labougle y Zozaya, 1986) y por el amplio conocimiento que se tiene de su flora apícola (Wulfrath y Speck, 1953; Espina y Ordetx, 1983). No obstante, a fin de poder caracterizar el origen botánico de la miel de Apis mellífera, no son suficientes las observaciones de campo, ya que no es posible la validación de que alguna miel pueda provenir de una planta o cultivo especifico solo porque éste se encuentra contiguo al apiario (Barth, 1970). En el presente trabajo melisopalinológico se establece el origen botánico de algunas mieles del estado de Guerrero, caracterizadas como oligoflorales y multiflorales, resultados que reflejan su riqueza florística. Se pretende que esta investigación palinológica despierte el interés de los apicultores para que visualicen la necesidad de este tipo de estudios y se implementen en otras regiones apícolas del estado de Guerrero, a fin de lograr la caracterización de la miel en todo el estado y poder incrementar su valor agregado en el mercado nacional e internacional.

\section{Agradecimientos}

Nuestro más sincero agradecimiento a los apicultores de la región Centro y Norte de Guerrero, a Liliana Cervantes
Martínez (Facultad de Ciencias, UNAM) por el apoyo en la determinación taxonómica del material botánico y a Margarita Reyes Salas (Instituto de Geología, UNAM) por las fotografías de polen en el Microscopio Electrónico de Barrido. Muchas gracias al Instituto de Geología de la Universidad Nacional Autónoma de México por las becas otorgadas a Nadia Ramírez Maldonado. Los autores agradecen a los revisores anónimos por sus valiosos comentarios y recomendaciones, así como a Sonia Helen Ponce Wainer por la revisión del resumen en inglés.

\section{Literatura citada}

Acosta-Castellanos S., Quiroz-García L., Arreguín-Sánchez M. de la L. y Fernández-Nava R. 2011. Análisis polínico de tres muestras de miel de Zacatecas, México. Polibotánica 32:171191.

Alfaro-Bates R.G., González-Acereto J.A., Ortíz-Díaz .J.J., Viera-Castro F.A., Burgos-Pérez A.I., Martínez-Hernández E. y Ramírez-Arriaga E. 2010. Caracterización palinológica de las mieles de la península de Yucatán. Universidad Autónoma de Yucatán y Comisión Nacional para el Conocimiento y Uso de la Biodiversidad, Mérida.

Alvarado J.L. y Delgado R.M. 1985. Flora apícola de Uxpanapa, Veracruz, México. Biótica 10:257-275.

Balleza J. de J. y Villaseñor J.L. 2002. La familia Asteraceae en el estado de Zacatecas (México). Acta Botanica Mexicana 59:5-69.

Barth O.M. 1970. Análise microscópica de algumas amostras de mel. 1 -. Pólen dominante. Anais do Academia Brasileiro de Ciência 42:351-366.

Cárdenas-Chávez S. 1985. Caracterización del ciclo apícola y flora nectarífera y polinífera, en la Chontalpa Tabasco, México. Tesis de Licenciatura, Colegio Superior de Agricultura Tropical, Cárdenas, Tabasco, 120 pp.

Castellanos-Potenciano B.P. 2010. Caracterización polínica estacional de miel inmadura de Apis mellifera L. en el estado de Tabasco. Tesis de Maestría en Ciencias, Colegio de Postgraduados, Institución de Enseñanza e Investigación en Ciencias Agrícolas, Campus Tabasco, México. 114 pp.

Castellanos-Potenciano B.P., Ramírez-Arriaga E. y Zaldívar-Cruz S.C. 2012. Análisis del contenido polínico de mieles producidas por Apis mellifera L. (Hymenoptera: Apidae) en el estado de Tabasco, México. Acta Zoológica Mexicana 28:13-36.

Crane E. 1975. Honey, a Comprehensive Survey. Heinemann, Londres.

Dávila P. y Sánchez-Ken J. 1994. Flora del Valle de TehuacánCuicatlán. Fascículo 3. Poaceae. Instituto de Biología, Universidad Nacional Autónoma de México, México D.F.

Díaz C.E. 2008. Estudio palinológico y fisicoquímico de la miel de Apis mellifera L. del municipio de San Pedro Tapanatepec, Oaxaca. Tesis de Licenciatura, Escuela de Medicina Veterinaria y Zootecnia, Universidad Autónoma "Benito Juárez de Oaxaca”, Oaxaca, 91pp.

Downey G., Hussey K., Kelly D.J., Walshe T.F. y Martin P.G. 2005. Preliminary contribution to the characterization of artisanal honey produced on the island of Ireland by palynological and physic-chemical data. Food Chemistry 91:347-354. 
Erdtman G. 1960. The acetolysis method; a revised description. Svensk Botanisk Tidskrift 54:561-564.

Espina-Pérez D. y Ordetx G.S. 1983. Flora Apícola Tropical. Editorial Tecnológica de Costa Rica, Cartago.

GEG [Gobierno del Estado de Guerrero]. 2016a. Nomenclaturas de los municipios del Estado de Guerrero. México.< http://guerrero.gob.mx/municipios/(consultado febrero, 2016).

GEG [Gobierno del Estado de Guerrero]. 2016b. < http://guerrero. gob.mx/municipios/centro/tixtla-de-guerrero/> (consultado 8 febrero 2016).

GEG [Gobierno del Estado de Guerrero]. 2016c. < http://guerrero. gob.mx/municipios/norte/teloloapan/> (consultado 8 febrero 2016).

Grimm E.C. 2011. Tilia Software, version 1.7.16, Illinois State Museum, Springfield IL.

INEGI [Instituto Nacional de Estadística, Geografía e Informática]. 2005. Información geográfica del estado de Guerrero. México. <http://www3.inegi.org.mx/sistemas/mexicocifras/default.aspx? $=12>$ (consultado 24 octubre 2005).

INEGI. 2011. Anuario estadístico de Guerrero. INEGI y Gobierno del Estado de Guerrero. Aguascalientes.

Labougle R.J.M. y Zozaya R.A. 1986. La apicultura en México. Ciencia y Desarrollo 12:17-36.

Lobreau-Callen D. y Callen G. 1982. Quelle est la composition pollinique d'un miel exotique? I. Bulletin de la Societé Versaillaise de Sciences Naturelles, Série 4 9:70-85.

Lot A. y Chiang F. 1986. Manual de Herbario. Administración y Manejo de Colecciones Técnicas de Recolección y Preparación de Ejemplares Botánicos. Instituto de Biología/Consejo Nacional de la Flora de México, A.C., México D.F.

Louveaux J., Maurizio A. y Vorwohl G. 1978. Methods of melissopalynology. Bee World 59:139-157.

Martínez-Hernández E., Cuadriello-Aguilar J.I, Téllez-Valdez O., Ramírez-Arriaga E., Sosa-Nájera Ma.S., Melchor-Sánchez J. E., Mediana-Camacho M. y Lozano-García Ma. del S. 1993. Atlas de las plantas y el polen utilizado por las cinco especies principales de abejas productoras de miel en la región del Tacana, Chiapas, México. Instituto de Geología, Universidad Nacional Autónoma de México, México D.F.

Martínez-Hernández E. y Ramírez Arriaga E. 1998. La importancia comercial del origen botánico de las mieles por medio de su contenido de granos de polen (Melisopalinología). Apitec 10:27-30.

Navarro C.L.A. 2008. Estudio palinológico y fisicoquímico de la miel de Apis mellifera L., en la Región costa de Oaxaca: Distritos Jamiltepec, Juquilla y Pochutla. Tesis de Licenciatura, Escuela de Medicina Veterinaria y Zootecnia, Universidad Autónoma "Benito Juárez de Oaxaca, 108 pp.

Olivera L., Ludlow W.B. y Fonseca R.M. 1998. Flora Palinológica de Guerrero. No. 7. Anacardiaceae. Facultad de Ciencias, Universidad Nacional Autónoma de México, México D.F.

Olvera-Luna A.R, Gama-López S. y Delgado-Salinas A. 2012. Flora del Valle de Tehuacán-Cuicatlán. Fascículo 107: Fabaceae. Instituto de Biología, Universidad Nacional Autónoma de México, México D.F.

Olvera M. 1997. Flora Palinológica de Guerrero. No. 5. Menyanthaceae, Nymphaeaceae, Pontederiaceae y Typhaceae. Facultad de Ciencias, Universidad Nacional Autónoma de México, México D.F.

Ortiz P.L. 1990. Contribución al conocimiento de la flora apícola gaditiana. Lagascalia 16:199-210.
Pacheco-Palomo K.G. 2011. Aspectos ecológicos y caracterización de la miel de Melipona beecheii Bennett 1831 (Apidae: Meliponinae), durante la época de lluvias (mayo-octubre de 2008), en China, Campeche. Tesis de Licenciatura, Universidad Autónoma de Campeche. Campeche, 92 pp.

Palacios-Chávez R., Ludlow-Wiechers y Villanueva R. 1991. Flora Palinológica de la Reserva de la Biosfera de Sian Ka'an, Quintana Roo, México. Centro de Investigaciones de Quintana Roo, Chetumal.

Piedras-Gutiérrez B. y Quiroz-García D.L. 2007. Estudio melisopalinológico de dos mieles de la porción sur del Valle de México. Polibotánica 23:57-75.

Pielou E.C. 1977. Mathematical Ecology. John Wiley \& Sons, Nueva York.

Punt W., Hoen P.P., Blackmore S., Nilsson S. y Le Thomas A. 2007. Glossary of pollen and spore terminology. Review of Palaeobotany and Palynology 143:1-81.

Quiroz-García D.L y Palacios-Chávez R. 1999. Determinación palinológica de los recursos florales utilizados por Centris inermis Friese (Hymenoptera: Apidae) en Chamela, Jalisco, México. Polibotánica 10:59-72.

Quiroz-García D.L. y Arreguín-Sánchez Ma. de la L. 2008. Determinación palinológica de los recursos florales utilizados por Apis mellifera L. (Hymenoptera: Apidae) en el estado de Morelos, México. Polibotánica 26:159-173.

Quiroz-García D.L., Arreguín-Sánchez Ma. de la L., FernándezNava R. y Martínez-Hernández E. 2011. Patrones estacionales de utilización de recursos florales por Scaptotrigona hellwegeri en la Estación de Biología Chamela, Jalisco, México. Polibotánica 31:89-119.

Ramalho M., Imperatriz-Fonseca V.I., Kleinert-Giovannini A. y Cortapassi-Laurino M. 1985. Exploitation of floral resources by Plebeia remota Holmberg (Apidae, Meliponinae). Apidologie 16:307-330.

Ramírez-Arriaga E., Martínez-Hernández E., Cuadriello-Aguilar E. y Lozano-García S. 1995. Estrategias de pecoreo de Plebeia sp. (Apidae), basado en el análisis melisopalinológico y en parámetros ecológicos en Chiapas. Implicaciones Evolutivas. En: Investigaciones Recientes en Paleobotánica y Palinología, Serie Arqueología, pp 113-154, Instituto Nacional de Antropología e Historia, México D.F.

Ramírez-Arriaga E. y Martínez-Hernández E. 1998. Resources foraged by Euglossa atroveneta (Apidae: Euglossinae) at Unión Juárez, Chiapas, México. A palynological study of larval feeding. Apidologie 29:347-359.

Ramírez-Arriaga E. y Martínez-Hernández E. 2007. Melissopalynological characterization of Scaptotrigona mexicana Gúerin (Apidae: Meliponini) and Apis mellifera L. (Apidae: Apini). honey samples in northern Puebla state, México. Journal of Kansas Entomological Society 80:377-391.

Ramírez-Arriaga E. 1989. Explotación de los recursos florales por Plebeia sp. (Apidae) en dos zonas con diferente altitud y vegetación en el Soconusco, Chiapas. Tesis Licenciatura, Facultad de Ciencias, Universidad Nacional Autónoma de México, México D.F. 159 p.

Ramírez-Arriaga E., Navarro-Calvo L.A. y Díaz-Carbajal E. 2011. Botanical characterization of Mexican honeys from a subtropical region (Oaxaca) based on pollen analysis. Grana 50:40-45.

Rivero-Montes L., Peñalba-Garmendia M.C., Ramírez-Olivas R. 
y Sau-Navarro M. A. 2002. La miel, mercado abierto. Ciencia y Desarrollo 28:30-38.

Rodríguez de la Cruz D., Sánchez-Reyes E., Sánchez-Durán S. y Sánchez-Sánchez J. 2013. Análisis palinológico de mieles comerciales monoflorales. Botanica Complutensis 37:171-180.

SAGARPA [Secretaría de Agricultura, Ganadería, Desarrollo Rural, Pesca y Alimentación]. 2009. La apicultura mexicana conquista mercados internacionales. < http://calderon.presidencia.gob.mx/infografias/2009/11/12/apicultura/index.html> (consultado 20 septiembre 2010).

SAGARPA. 2013. < http://www.sagarpa.gob.mx/ganaderia/Documents/2015/APICULTURA/Notiabj\%202015-1.pdf > (consultado 18 enero 2013).

Sancho M.T., Muniategui S., Huidobro J.F. y Simal J. 1991. Mieles del país Vasco. VI: Elementos de mielada. Anales de Bromatología XLIII-2/3:165-172.

Seaby R.M.H. y Henderson P.A. 2007. Species Diversity and Richness IV. Measuring and understanding biodiversity. Pisces Conservation, Hampshire.

Shannon C.E. y Weaver W. 1949. The mathematical theory of communication. University of Illinois Press, Urbana.

Valencia A.S. 2004. Diversidad del género Quercus (Fagaceae) en México. Boletín de la Sociedad Botánica de México 75:33-53.

Vargas A. y Ludlow-Wiechers B. 1993. Flora Palinológica de Guerrero. No. 3. Araliaceae. Facultad de Ciencias, Universidad Nacional Autónoma de México. México D.F.

Vázquez S. y Martínez-Mena A. 1991. Flora Palinológica de Guerrero. No. 1. Onagraceae. Facultad de Ciencias, Universidad
Nacional Autónoma de México, México D.F.

Villanueva G. R. 1984. Plantas de importancia apícola en el ejido de Plan del Río, Veracruz, México. Biótica 9:279-340.

Villanueva-G R. 2002. Polliniferous plants and foraging strategies of Apis mellifera (Hymenoptera: Apidae) in the Yucatán Peninsula, Mexico. Revista de Biología Tropical 50:1035-1043.

Villaseñor J.L. 2003. Diversidad y distribución de las Magnoliophyta de México. Interciencia 28:160-167.

Villegas-Durán G., Cajero A., Bolaños A., Miranda S., Pérez M., Guzmán Q., Tah B., Osorno L. y Sánchez R. 1998. Flora Nectarífera y Polinífera en la Península de Yucatán. Secretaría de Agricultura, Ganadería, Desarrollo Rural, Pesca y Alimentación, México D.F.

Villegas-Durán G., Bolaños-Medina A., Miranda-Sánchez J.A., Sandoval H.R. y Lizama M.J.M. 2003. Flora nectarífera y polinífera en el estado de Veracruz. Secretaria de Agricultura, Ganadería, Desarrollo Rural, Pesca y Alimentación, México D.F.

Villegas-Durán G., Bolaños M.A., Miranda S.J.A. y González Q.R. 2002. Flora nectarífera y polinífera en el estado de Guerrero. Secretaría de Agricultura, Ganadería Desarrollo Rural, Pesca y Alimentación, México D.F.

Villegas-Durán G., Bolaños M.A., Miranda S.J.A. Quintana, R.I.L., Guzmán Q.E.O. y Zavala, R.J.J.M. 1999. Flora nectarífera y polinífera en el estado de Michoacán. Secretaría de Agricultura, Ganadería, Desarrollo Rural, Pesca y Alimentación, México D.F.

Wulfrath A. y Speck J.J. 1953. La Flora melífera. En: Enciclopedia Apícola, Agrícola Mexicana, México D.F.

Recibido: 19 de agosto de 2014

Aceptado: 10 de septiembre de 2014 